\title{
Una identidad terremoteada. Comunidad y territorio en el Chile de 1960*
}

Bárbara Silva Avaria, Alfredo Riquelme Segovia

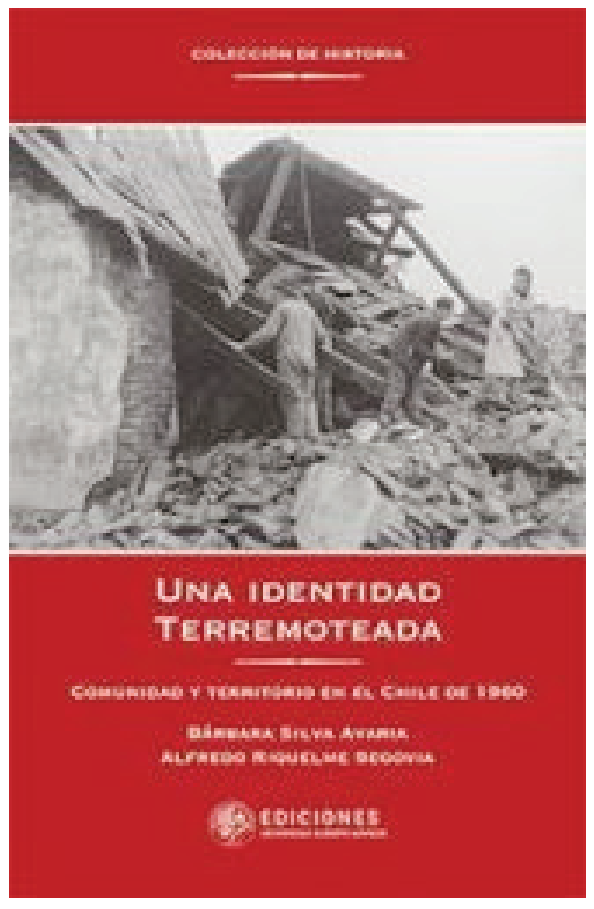

Diego Arango-López

La construcción de las identidades nacionales se relaciona con múltiples factores sociales, culturales, económicos, políticos, institucionales y territoriales. El libro de Bárbara Silva Avaria y Alfredo Riquelme Segovia,

* 2018 Santiago: Ediciones Universidad Alberto Hurtado. 185 páginas. 
aborda el proceso de construcción y refuerzo de la identidad nacional chilena en 1960 con relación a la celebración del sesquicentenario de la independencia y al terremoto de Valdivia del mismo año. El propósito del trabajo de Silva y Riquelme es "(...) revisitar la relación entre política y cultura en la construcción de la nación en Chile, integrando una perspectiva telúrica, la que se asocia íntimamente con la historia de la nación" (Silva y Riquelme 2018: 19). En ese sentido, se propone una lectura en tres capítulos en los cuales se abordan las dimensiones política, simbólica y territorial de la noción de identidad nacional en Chile en 1960.

Los tres capítulos del libro plantean una aproximación a la identidad nacional que los autores retoman del concepto de comunidad imaginada desarrollado por Benedict Anderson (2003). Así, plantean que, además de las perspectivas materiales ofrecidas por el territorio, éste contiene en sí connotaciones simbólicas que dialogan en el proceso de construcción de la identidad nacional. De esta manera, el planteamiento se enfoca en dos acontecimientos, el sesquicentenario y el terremoto, ocurridos en 1960 alrededor de los cuales los autores articulan su argumentación.

El primer acontecimiento, refiere a la conmemoración de los 150 años de la independencia chilena. Dicha celebración, tal como lo muestran los autores, es interpretada como un punto medio entre el centenario y el bicentenario, en el cual, más que resaltar la equidistancia temporal, se señala la importancia de un acontecimiento programado que marca diferencias respecto de las representaciones de la identidad nacional del centenario y del bicentenario. Ahora bien, este acontecimiento es acompañado de un evento inesperado, el terremoto de Valdivia 1960, que según Silva y Riquelme demuestra la importancia de la condición telúrica en la construcción de la identidad nacional chilena. Así, se establece que, tanto el sesquicentenario como el terremoto, son eventos que se entremezclan en el proceso simbólico de construcción de una comunidad imaginada.

Por una parte, hay que señalar que la propuesta de articulación del desastre y de la condición telúrica dentro del sistema de representación sobre el cual se construye la identidad nacional de mediados de siglo es una apuesta interdisciplinar fundamental. Pues, incorpora el elemento territorial en la noción de identidad mediante un ejercicio historiográfico riguroso que utiliza herramientas del análisis geográfico. Ahora bien, por otra parte, los autores no reparan en la necesidad de interpretar 
el desastre como un acontecimiento especial o una categoría con una singularidad conceptual que sobrepasa su condición espacial y territorial. Es decir, el terremoto es visto como un acontecimiento geográfico que caracteriza el territorio nacional, pero no es interpretado como un evento espacial distinto, marcado por la sorpresa, por su capacidad destructiva, o por su caracterización como riesgo extensivo o factor de vulnerabilidad en el territorio chileno. De esta manera, el desastre, se asemeja a otros acontecimientos territoriales no destructivos, que quizás sirvan para explicar y describir la capacidad de los grupos sociales de relacionarse con sus espacios. Por lo tanto, a partir del libro, queda sin resolver la pregunta sobre la interpretación de los desastres en el ejercicio historiográfico, puesto que en este caso son vistos a través de su condición territorial, pero sin incorporar su especificidad como eventos socio-naturales especialmente asociados a nociones como la vulnerabilidad, el riesgo, la amenaza, el peligro y la seguridad.

En ese sentido, los autores plantean que la celebración del sesquicentenario, con todo el desarrollo simbólico que implica, coincide con el terremoto que cumple la función de reforzar una idea de nación de larga trayectoria en el imaginario chileno, la de la nación telúrica. No obstante, también podría argumentarse, con base en las mismas fuentes, que el carácter destructivo del terremoto irrumpe en esa construcción simbólica de la nación generando la necesidad de replantear una serie de postulados sobre la nación chilena, que como bien lo señalan los autores, refieren a conceptos e ideas relativamente desconectadas del ethos popular y referentes a los deseos de grandeza de unas elites oligárquicas.

Ahora bien, lo que sí queda claro más allá de cualquier diferencia de interpretación, es que la comunidad nacional en su conjunto, por la coincidencia de ambos eventos, se ve en la ineludible necesidad de incorporar el factor territorial y telúrico en su proceso de construcción de identidad nacional. Sin embargo, al interpretar el terremoto como el refuerzo de un proceso que, por la recurrencia de los movimientos de tierra, estaba en curso desde tiempos anteriores, se reduce asimismo la particularidad del terremoto de Valdivia. Pues, si bien es cierto que se trata de un acontecimiento asociado a un proceso geológico no excepcional, la magnitud del terremoto del 60 , con sus $9,5^{\circ}$ en la escala de Richter, es la más alta desde que se tienen registros de la actividad sísmica en la historia. En ese sentido, desde el punto de vista de socio-cultural, la potencia del terremoto es en sí misma una característica diferenciadora 
que, desafortunadamente, no es abordada por los autores. Es decir, si bien queda demostrado que el carácter telúrico es efectivamente una característica que se incorpora al discurso de la identidad nacional, la especificidad de este terremoto, aún siendo el más potente y destructivo de la historia, no se constituye como un punto de inflexión en el proceso simbólico de construcción de la comunidad imaginada chilena.

El carácter telúrico de la identidad nacional, que como lo demuestran los autores, es parte de la construcción simbólica chilena, hace referencia a los terremotos como algo catastrófico o negativo. Es decir, el terremoto es algo a lo cual se debe resistir y que requiere de una recuperación posterior. De cierta forma, la interpretación del terremoto que observan los autores a través de una amplia, rigurosa y sistemática revisión de fuentes, en especial de prensa, parece demostrar que la identidad telúrica se construye con base en una dialéctica negativa. Dicho de otra manera, la chilenidad no se logra de la mano de la condición telúrica sino a pesar de ella. Así, esta identidad chilena entraría en conflicto con conceptos, que se están desarrollando en esa época, como la mitigación y prevención del riesgo. Pues, el orgullo chileno no reside en la capacidad de la sociedad para armonizar sus formas de vida con el movimiento de la tierra sino, por el contrario, en la capacidad de temer el temblor y recuperarse después de sufrir sus consecuencias destructivas. Así, como se resalta en el libro, el ser chileno se asocia con la capacidad de los integrantes de la comunidad imaginada para "renacer" o para "solidarizar" más no con el establecimiento progresivo de una simbiosis entre lo físico y lo cultural.

Ahora bien, algunos argumentos presentados en el tercer capítulo parecen desafiar esta presentación del terremoto como un evento sufrido por los chilenos y del cual se recuperan posteriormente. Pues, se resaltan discursos referentes a la solidaridad que, más que exaltar el carácter altruista del chileno ante su compatriota caído en desgracia, exigen el desarrollo de mecanismos de mitigación del riesgo, bien sea desde lo militar o desde el aparato ejecutivo. Lo cual plantea una interesante reflexión sobre la responsabilidad gubernamental en caso de desastre, tanto a nivel nacional como internacional, estableciendo, aun sin citarlo, posibles diálogos con el trabajo de Revet y Langumier (2013) sobre el gobierno internacional de los desastres.

Así, el libro ofrece una perspectiva sobre lo que se construyó en términos identitarios gracias a dos acontecimientos vividos en el Chile de 1960, 
el sesquicentenario y el terremoto. Se presenta un análisis que logra incorporar el carácter geográfico con el análisis social, político y cultural sin caer en falsos determinismos. En ese sentido, si bien los autores plantean que el recuerdo de 1960 quedó profundamente marcado por el terremoto, el ejercicio que realizan no se recorre las fuentes de las décadas posteriores para demostrar dicho planteamiento. No obstante, dicha afirmación sirve para abrir una valiosa veta de investigaciones, pues plantea la necesidad de recorrer las fuentes de la historia reciente, explorando las décadas del 70, 80, 90, 2000 y 2010, para analizar cómo es incorporado el desastre de 1960 en la memoria colectiva (Halbwachs 1950) chilena.

\section{Bibliografía}

Anderson, B. 2003. Comunidades Imaginadas. México: Fondo de Cultura Económica.

Halbwachs, M. 1967 (1950) La mémoire collective. París: Presses Universitaires de France.

Revet, S., Langumier, J. 2013. Le gouvernement des catastrophes. Paris: Karthala. 
